\title{
The Year in Science and Medicine
}

\author{
Ze'ev Hochberg
}

\section{New therapy: glue the fragile parts}

\section{Identification of small molecules rescuing fragile $X$ syndrome phenotypes in Drosophila}

\author{
Chang S, Bray SM, Li Z, Zarnescu DC, He C, Jin P, Warren ST \\ Department of Human Genetics, Emory University School of Medicine, Atlanta, Ga., USA \\ Nat Chem Biol 2008;4:256-263
}

Context: Fragile $\mathrm{X}$ syndrome is caused by the functional loss of the fragile $\mathrm{X}$ mental retardation 1 (FMR1) gene. Deletion of the FMR1 ortholog in Drosophila (Fmr1) recapitulates many phenotypes associated with fragile X syndrome. Fmr1 mutant Drosophila die during development when reared on food containing increased levels of glutamate, which is consistent with the theory that FMR1 loss results in excess glutamate signaling.

Methods: Using this lethal phenotype, this study screened a chemical library of 2,000 compounds.

Results: They identified nine molecules that rescued the lethality, including three that implicate the GABAergic inhibitory pathway. Indeed, GABA treatment rescued several known Fmr1 mutant phenotypes in flies, including mushroom body defects, excess Futsch translation and abnormal male courtship behavior. In addition, the screen reveals that the muscarinic cholinergic receptors may have a role in fragile X syndrome in parallel to the GABAergic pathway.

Conclusion(s): These data are consistent with GABAergic inhibition of the enhanced excitatory pathway in fragile $\mathrm{X}$ syndrome. These results point to potential therapeutic approaches for treating fragile $\mathrm{X}$ syndrome.

\begin{abstract}
Fragile $\mathrm{X}$ syndrome is a common cause of mental retardation in humans, and the Drosophila study might have discovered a therapeutic target to treat it. Fragile $X$ syndrome is caused by loss of the FMRP translational regulator. A current hypothesis proposes that FMRP functions downstream of glutamate receptor signaling to regulate synaptic connections. Null drosophila Fmr1 mutants display increased presynaptic structural complexity and elevated presynaptic vesicle pools, which are rescued by blocking glutamate receptor signaling. These data show that glutamate and dFMRP convergently regulate presynaptic properties [1]. The fruit flies lacking the fragile X-equivalent gene Fmr1 exhibit some physical and behavioral features of fragile $X$ syndrome, but in addition have several dysmorphic features, and glutamate kills mutant embryos. GABA relieved glutamate toxicity, and restored several features, including normal courtship behavior and some brain morphology.
\end{abstract}

\section{Lesson from our past: adapt your colors}

\section{First Homo erectus from Turkey and implications for migrations into temperate Eurasia}

Kappelman J, Alcicek MC, Kazanci N, Schultz M, Ozkul M, Sen S

Department of Anthropology, The University of Texas, Austin, Tex., USA

Am J Phys Anthropol 2008;135:110-116

Context: Remains of fossil hominins from temperate regions of the Old World are rare across both time and space, but such specimens are necessary for understanding basic issues in human evolution including 
linkages between their adaptations and early migration patterns. This report describes the remarkable circumstances surrounding the discovery of the first fossil hominin calvaria from the Denizli province of western Turkey. The new specimen fills an important geographical and temporal gap and displays several anatomical features that are shared with other Middle Pleistocene hominins from both Africa and Asia attributed to Homo erectus.

Results: The calvaria preserves an unusual pathology on the endocranial surface of the frontal bone that is consistent with a diagnosis of Leptomeningitis tuberculosa (TB), and this evidence represents the most ancient example of this disease known for a fossil human.

Conclusion(s): TB is exacerbated in dark-skinned peoples living in northern latitudes by a vitamin D deficiency because of reduced levels of ultraviolet radiation (UVR). Evidence for TB in the new specimen supports the thesis that reduced UVR was one of the many climatic variables presenting an adaptive challenge to ancient hominins during their migration into the temperate regions of Europe and Asia.

We knew of Homo erectus leaving in East Africa and Asia. The present specimen from Turkey lived 500,000 year ago in a warm interglacial stage that was preceded and followed by two of the coldest glacial stages of the past million years. As hominines ventured out of Africa they received significantly less UV radiation, and their skin depigmented to a degree that permitted UV-induced synthesis of previtamin D3 in northern or southern latitudes, where UV radiation is less intense. It is now widely appreciated that vitamin $\mathrm{D}$ has a wide variety of noncalcemic functions, including an immunoregulatory role. We have recently learned about the innate antimicrobial mechanism by which the activation of Toll-like receptors produces the enzyme Cyp27B1 which catalyzes active vitamin D3 that in turn triggers the induction of cathelicidin mRNA that kills Mycobacterium tuberculosis. The fossils found in Turkey had bone findings which are consistent with TB. The link between a vitamin D deficiency in dark-skinned peoples living at high latitude and TB has been raised before in, for example, a high TB incidence in a Gujarati Asian population living in London, and in native Senegalese troops that increased with the amount of time that the battalion was barracked in France during WWI, and a TB infection rate 68 times higher for Somali immigrants living in Minnesota than the national Caucasian rate. You may recall that increased exposure to sunlight was one of the most widely accepted treatment regimens for TB in past centuries.

\section{New hope - Repair self}

\section{Reprogramming of human somatic cells to pluripotency with defined factors}

Park IH, Zhao R, West JA, Yabuuchi A, Huo H, Ince TA, Lerou PH, Lensch MW, Daley GQ

Division of Pediatric Hematology/Oncology, Children's Hospital Boston and Dana Farber Cancer Institute, Boston,

Mass., USA

Nature 2008;451:141-146

Context: Pluripotency pertains to the cells of early embryos that can generate all of the tissues in the organism. Recently, murine fibroblasts have been reprogrammed directly to pluripotency by ectopic expression of four transcription factors to yield induced pluripotent stem (iPS) cells.

Methods: Using these same factors, this study derived iPS cells from fetal, neonatal and adult human primary cells, including dermal fibroblasts isolated from a skin biopsy of a healthy research subject.

Results: Human iPS cells resembled embryonic stem cells in morphology and gene expression and in the capacity to form teratomas in immune-deficient mice.

Conclusion(s): These data demonstrate that defined factors can reprogram human cells to pluripotency, and establish a method whereby patient-specific cells might be established in culture.

Applying four defined transcription factors to cells from adult human tissue, this group generated at a relatively high frequency $(0.1 \%)$ induced pluripotent stem (iPS) cells with all the properties and the same developmental potential as embryonic stem cells. In addition to the murine genes that have previously been reported, Park et al. introduced two additional immortalizing and transforming factors: 
hTERT (which encodes the catalytic subunit of human telomerase enzyme) and the gene for SV40 large $T$ antigen. They conclude that it may be possible now to establish patient-specific cells from one's own fibroblasts. The generation of iPS cells through direct reprogramming avoids the ethical controversies surrounding the use of embryos for deriving stem cells. It remains to be proven that the reprogramming did not induce other undetected genetic changes.

\section{New mechanism: direct feminine attack}

\section{DNA oxidation as triggered by H3K9me2 demethylation drives estrogen-induced gene expression}

Perillo B, Ombra MN, Bertoni A, Cuozzo C, Sacchetti S, Sasso A, Chiariotti L, Malorni A, Abbondanza C, Avvedimento EV

Istituto di Scienze dell'Alimentazione, Consiglio Nazionale delle Ricerche (CNR), Avellino, Italy

Science 2008;319:202-206

Context: Modifications at the $\mathrm{N}$-terminal tails of nucleosomal histones are required for efficient transcription in vivo.

Methods: This study analyzed how H3 histone methylation and demethylation control expression of estrogen-responsive genes.

Results: DNA-bound estrogen receptor directs transcription by participating in bending chromatin to contact the RNA polymerase II recruited to the promoter. This process is driven by receptor-targeted demethylation of $\mathrm{H} 3$ lysine 9 at both enhancer and promoter sites and is achieved by activation of resident LSD1 demethylase. Localized demethylation produces hydrogen peroxide, which modifies the surrounding DNA and recruits 8-oxoguanine-DNA glycosylase 1 and topoisomerase-II $\beta$, triggering chromatin and DNA conformational changes that are essential for estrogen-induced transcription.

Conclusion(s): The data show a strategy that uses controlled DNA damage and repair to guide productive transcription.

Estrogens regulate transcription of target genes by binding to cognate estrogen receptors (ER $\alpha$ and $E R \beta)$, which then bind with high affinity to estrogen-responsive DNA elements (EREs). But there is more to it; after hormone activation, estrogens modify epigenetic signatures. ER $\alpha$ interacts with transcription co-activators that covalently modify histone proteins within nucleosomes and contribute to the control of gene expression. This paper shows that transcription of estrogen-responsive genes is driven by hormone-dependent demethylation of a critical lysine in histone $\mathrm{H} 3$ to induce a local oxidative burst that modifies the surrounding DNA by guanine oxidation, which is subsequently removed by specific glycosylases. The RNA pol II complex intimately associates with DNA repair enzymes to initiate estrogen-dependent gene expression.

Concepts revised: why elephants have such a long memory

\section{Epigenetic memory of an active gene state depends on histone H3.3 incorporation into chromatin in the absence of transcription}

Ng RK, Gurdon JB

Wellcome Trust/Cancer Research UK Gurdon Institute, Cambridge, UK

Nat Cell Biol 2008;10:102-109

Context: The remarkable stability of gene expression in somatic cells is exemplified by the way memory of an active gene state is retained when an endoderm cell nucleus is transplanted to an enucleated egg. Aims: Analysis $t$ of the mechanism of a similar example of epigenetic memory. 
Results: The study finds that memory can persist through 24 cell divisions in the absence of transcription and applies to the expression of the myogenic gene $M y o D$ in non-muscle cell lineages of nuclear transplant embryos. It shows that memory is not explained by the methylation of promoter DNA, but correlates with the association of histone $\mathrm{H} 3.3$ with the MyoD promoter in embryos that display memory but not in those where memory has been lost. The association of a mutated histone H3.3 (H3.3 E4, which lacks the methylatable H3.3 lysine 4) with promoter DNA eliminates memory, indicating a requirement of H3.3 K4 for memory. They also show that overexpression of H3.3 can enhance memory in transplanted nuclei.

Conclusion(s): The association of histone $\mathrm{H} 3.3$ with the $M y_{\mathrm{O}} \mathrm{D}$ promoter makes a necessary contribution to this example of memory. Hence, they suggest that epigenetic memory helps to stabilize gene expression in normal development; it might also help to account for the inefficient reprogramming in some transplanted nuclei.

Differentiated cells 'remember' their uniqueness even when they move to a new cellular environment. This study shows that histone H3.3, which is enriched in actively expressed genes, is required for the persistence of some active gene states. The results show that epigenetic memory is remarkably stable after two rounds of nuclear transfer and 24 mitoses, and in the absence of transcription. In search of a mechanism for this memory, the authors show that DNA methylation had no effect on expression, and that the mechanism has to do with histone H3.3 levels; overexpressing wild-type H3.3 in nuclear transfer embryos enhanced memory and overexpression of a mutant form led to memory reduction. This type of memory occurs in cells that are committing to a developmental pathway, but not at loci involved in terminal differentiation. The authors conclude that 'the stable inheritance of cellular memory might provide an explanation for the decreasing success of nuclear transfer from more differentiated donor cell nuclei'.

\section{Wonder of the year: more than just technology}

\section{Staged in vitro reconstitution and implantation of engineered rat kidney tissue}

Rosines E, Sampogna RV, Johkura K, Vaughn DA, Choi Y, Sakurai H, Shah MM, Nigam SK

Departments of Pediatrics, Medicine, Bioengineering, and Cellular and Molecular Medicine, University of California at San Diego, La Jolla, Calif., USA

Proc Natl Acad Sci USA 2007; 104:20938-20943

Context: A major hurdle for current xenogenic-based and other approaches aimed at engineering kidney tissues is reproducing the complex three-dimensional structure of the kidney.

Methods: Here, a stepwise, in vitro method of engineering rat kidney-like tissue capable of being implanted is described. Based on the fact that the stages of kidney development are separable into in vitro modules, an approach was devised that sequentially induces an epithelial tubule (the Wolffian duct) to undergo in vitro budding, followed by branching of a single isolated bud and its recombination with metanephric mesenchyme.

Results: Implantation of the recombined tissue results in apparent early vascularization. Optimization studies (of growth factor and matrix) indicate multiple suitable combinations and suggest both a most robust and a minimal system. A whole-genome microarray analysis suggested that recombined tissue recapitulated gene expression changes that occur in vivo during later stages of kidney development, and a functional assay demonstrated that the recombined tissue was capable of transport characteristic of the differentiating nephron. The approach includes several points where tissue can be propagated.

Conclusion(s): In principle, an unbranched epithelial tubular structure (potentially constructed from cultured cells) can be induced to form kidney tissue such that this in vitro engineered tissue is capable of being implanted in host rats and developing glomeruli with evidence of early vascularization. The data also show how functional, 3D kidney tissue can assemble by means of interactions of independent modules separable in vitro, potentially facilitating systems-level analyses of kidney development. 
To engineer a specialized tissue like the kidney, this study broke the process into a number of substeps, each with the potential for optimization and development. To entice the cells into the complex three-dimensional structure of a naturally functioning kidney, the authors employed growth factors and extracellular matrix components in a series of culture stages. First, ureteric buds (UBs) were grown from Wolffian duct microdissected from an embryonic rat. They then induced the UBs to fuse with metanephric mesenchyme into a structure resembling the late embryonic kidney, including apparent filtration units. The gene expression in the engineered tissue was similar, but not identical, to that in a natural kidney. The tissue survived implantation in inbred adult rats and seemed to develop a simple blood network, but whether it will function like normal kidney tissue remains to be seen. You must have heard this many times, 'A pastor, a doctor and an engineer were ...'. They now all merge in single laboratory.

\title{
Drool for fitness
}

\section{Diet and the evolution of human amylase gene copy number variation}

Perry GH, Dominy NJ, Claw KG, Lee AS, Fiegler H, Redon R, Werner J, Villanea FA, Mountain JL, Misra R, Carter NP, Lee $\mathrm{C}$, Stone AC

School of Human Evolution and Social Change, Arizona State University, Tempe, Ariz., USA

Nat Genet 2007;39:1256-1260

Context: Starch consumption is a prominent characteristic of agricultural societies and hunter-gatherers in arid environments. In contrast, rainforest and circum-arctic hunter-gatherers and some pastoralists consume much less starch. This behavioral variation raises the possibility that different selective pressures have acted on amylase, the enzyme responsible for starch hydrolysis.

Results: This study finds that copy number of the salivary amylase gene (AMY1) is correlated positively with salivary amylase protein level and that individuals from populations with high-starch diets have, on average, more AMY1 copies than those with traditionally low-starch diets. Comparisons with other loci in a subset of these populations suggest that the extent of AMY1 copy number differentiation is highly unusual.

Conclusion(s): This example of positive selection on a copy number-variable gene is one of the first discovered in the human genome. Higher AMY1 copy numbers and protein levels probably improve the digestion of starchy foods and may buffer against the fitness-reducing effects of intestinal disease.

Hunter-gatherers living in the rainforests or near the Arctic circle, have historically existed on a diet rich in protein and low in starch as compared to agricultural societies. This paper shows that the human genome adapted to such differences in the amount of dietary starch. AMY1 is one of the few genes that show extensive copy-number variation between individuals; extra AMY1 copies endow the individuals carrying them with the capacity to produce more salivary amylase. In terms of evolutionary fitness, digestion of starch during chewing may be vital in people likely to suffer from diarrheal diseases. One wonders to what extent our children, who are exposed to huge amounts of starch in their diet, have appropriate copy number of the AMY1 gene.

\section{Localizing recent adaptive evolution in the human genome}

\author{
Williamson SH, Hubisz MJ, Clark AG, Payseur BA, Bustamante CD, Nielsen R
}

Department of Biological Statistics and Computational Biology, Cornell University, Ithaca, N.Y., USA

sw292@cornell.edu

PLoS Genet 2007;3:e90

Context: Identifying genomic locations that have experienced selective sweeps is an important first step toward understanding the molecular basis of adaptive evolution.

Methods: Using statistical methods that account for the confounding effects of population demography, recombination rate variation, and single-nucleotide polymorphism ascertainment, while also providing fine-scale estimates of the position of the selected site, this paper analyzed a genomic dataset of 1.2 million 
human single-nucleotide polymorphisms genotyped in African-American, European-American, and Chinese samples.

Results: They identified 101 regions of the human genome with very strong evidence $\left(\mathrm{p}<10^{-5}\right)$ of a recent selective sweep, and where our estimate of the position of the selective sweep falls within $100 \mathrm{~kb}$ of a known gene. Within these regions, genes of biological interest include genes in pigmentation pathways, components of the dystrophin protein complex, clusters of olfactory receptors, genes involved in nervous system development and function, immune system genes, and heat shock genes. We also observe consistent evidence of selective sweeps in centromeric regions.

Conclusion(s): Recent adaptation is strikingly pervasive in the human genome, with as much as $10 \%$ of the genome affected by linkage to a selective sweep.

Natural selection has caused as much as $10 \%$ of the human genome to change in some populations in the last 15,000 to 100,000 years, when people began migrating out of Africa. This study looked for areas where most members of a population showed the same genetic changes. Thus, the skin pigmentation gene (we have only one - MC1R - that explains the entire colors spectrum), adapted to the new UVB levels. The study found no evidence of differences in genes that control brain development among the various geographical groups. We have previously reviewed the divergence of genes between a human and a chimpanzee [2]. But this study scanned genome sequences that compared many humans to each other throughout the entire genome, with new strict statistical methods that correct for many potential biases that creep into this kind of analysis. They identified 101 regions of the human genome with strong evidence of very recent selection. These regions include genes that control muscle proteins (muscular dystrophy gene), receptors that relate to hearing, genes involved in nervous system function and development, immune system genes, heat shock genes and lactase. The latter evolved as humans migrated and domesticated animals for tolerating milk throughout life.

\section{Improvement of water use efficiency in rice by expression of HARDY, an Arabidopsis drought and salt tolerance gene}

Karaba A, Dixit S, Greco R, Aharoni A, Trijatmiko KR, Marsch-Martinez N, Krishnan A, Nataraja KN, Udayakumar M, Pereira A

Plant Research International, Wageningen University and Research Centre, Wageningen, The Netherlands Proc Natl Acad Sci USA 2007;104:15270-15275

Context: Freshwater is a limited and dwindling global resource; therefore, efficient water use is required for food crops that have high water demands, such as rice, or for the production of sustainable energy biomass.

Results: The expression of the Arabidopsis HARDY (HRD) gene in rice improved water use efficiency, the ratio of biomass produced to the water used, by enhancing photosynthetic assimilation and reducing transpiration. These drought-tolerant, low-water-consuming rice plants exhibit increased shoot biomass under well-irrigated conditions and an adaptive increase in root biomass under drought stress. The HRD gene, an AP2/ERF-like transcription factor, identified by a gain-of-function Arabidopsis mutant hrd-D having roots with enhanced strength, branching, and cortical cells, exhibits drought resistance and salt tolerance, accompanied by an enhancement in the expression of abiotic stress associated genes. HRD overexpression in Arabidopsis produces thicker leaves with more chloroplast-bearing mesophyll cells, and in rice, there is an increase in leaf biomass and bundle sheath cells that probably contributes to the enhanced photosynthesis assimilation and efficiency.

Conclusion(s): The results exemplify application of a gene identified from the model plant Arabidopsis for the improvement of water use efficiency coincident with drought resistance in the crop plant rice.

Water scarcity, caused by the rapidly increasing world population and the accompanying increases in water use for social and economic development, threatens sustainable world crop production that consumes most of the global water resources. Rice uses 2-3 times more water than other food crops, while it is the primary source of food for more than half of the world's population. Plant resistance to drought stress can be improved through drought avoidance or drought tolerance. This report provides a way to achieve improved water use efficiency and drought resistance in rice. It remains for decision makers to be convinced by the scientific evidence and give a green light for Green Super Rice. 


\section{Toward a confocal subcellular atlas of the human proteome}

Barbe L, Lundberg E, Oksvold P, Stenius A, Lewin E, Bjorling E, Asplund A, Ponten F, Brismar H, Uhlen M, Andersson-Svahn $\mathrm{H}$

Department of Biotechnology, AlbaNova University Center, Royal Institute of Technology, Stockholm, Sweden Mol Cell Proteomics 2008;7:499-508

Background: Information on protein localization on the subcellular level is important to map and characterize the proteome and to better understand cellular functions of proteins.

Methods: This is a report on a pilot study of 466 proteins in three human cell lines aimed to allow large scale confocal microscopy analysis using protein-specific antibodies.

Results: Approximately 3,000 high-resolution images were generated, and more than $80 \%$ of the analyzed proteins could be classified in one or multiple subcellular compartment(s). The localizations of the proteins showed, in many cases, good agreement with the Gene Ontology localization prediction model.

Conclusion(s): This is the first large scale antibody-based study to localize proteins into subcellular compartments using antibodies and confocal microscopy. The results suggest that this approach might be a valuable tool in conjunction with predictive models for protein localization.

The Human Protein Atlas http://www.proteinatlas.org/ was launched in October 2007 as a comprehensive database that provides the protein expression profiles for a large number of human proteins, presented as immunohistological images from most human tissues. The initial version had over 3,000 proteins out of the human's $\sim 200,000$. An update was released in June 2008 with over 7,500 proteins, each identified with a specific antibody, and 1,200,000!!! figures. The new version displays more tissue information, more cell images have been added, and some cell types have got corrected names. This will become a working tool for many, but don't miss it even if only for curiosity.

References

1. Pan L, Woodruff E 3rd, Liang P, Broadie K: Mechanistic relationships between Drosophila fragile X mental retardation protein and metabotropic glutamate receptor A signaling. Mol Cell Neurosci 2008;37:747-760.

2. Hochberg Z, Carel J-C: The year in science and medicine; in Carel J-C, Hochberg Z (eds): Yearbook of Pediatric Endocrinology 2006. Basel, Karger, 2006, p 180. 
